\title{
Comparison of Kinetic Theory and Hydrodynamics for Poiseuille Flow
}

\author{
Yihao Zheng, ${ }^{1}$ Alejandro L. Garcia, ${ }^{2,3}$ and Berni J. Alder ${ }^{1,3}$
}

Received January 31, 2002; accepted June 24, 2002

Comparison of particle (DSMC) simulation with the numerical solution of the Navier-Stokes (NS) equations for pressure-driven plane Poiseuille flow is presented and contrasted with that of the acceleration-driven Poiseuille flow. Although for the acceleration-driven case DSMC measurements are qualitatively different from the NS solution at relatively low Knudsen number, the two are in somewhat better agreement for pressure-driven flow.

KEY WORDS: Kinetic theory; Boltzmann equation; Poiseuille flow; direct simulation Monte Carlo.

\section{INTRODUCTION}

The hydrodynamic equations may be obtained as a first approximation to the Boltzmann equation ${ }^{(1)}$ and are generally accurate at the macroscopic level except under some extreme conditions, ${ }^{(2,3)}$ since these Navier-Stokes equations, are derived under the assumptions of local equilibrium, small gradients and flows with small Knudsen number.

Surprisingly, even for the simple case of acceleration-driven Poiseuille flow with relatively small gradients and Knudsen number, the NavierStokes equations fail to give qualitatively correct predictions. Specifically, they fail to reproduce the central minimum in the temperature profile and a non-constant pressure profile, which are both predicted by kinetic theory

\footnotetext{
${ }^{1}$ Department of Applied Science, University of California, Davis, California 95616.

${ }^{2}$ Department of Physics, San Jose State University, San Jose, California 95192; e-mail: algarcia (a)algarcia.org

${ }^{3}$ Center for Applied Scientific Computing, Lawrence Livermore National Laboratory, Livermore, California 94551.
} 
and observed in numerical simulations down to Knudsen numbers of $10^{-2} \cdot{ }^{(4-11)}$ Furthermore, it is not possible to correct this failure by modifying the equation of state, transport coefficients or boundary conditions and, unlike slip phenomena, the discrepancy is not just near a boundary but throughout the system. The object of this paper is to investigate whether a similar discrepancy occurs in what appears to be an equivalent system, namely pressure-driven Poiseuille flow.

The Navier-Stokes equations have the following form: ${ }^{(12)}$

$$
\begin{aligned}
\frac{\partial \rho}{\partial t}+\nabla \cdot(\rho \mathbf{v}) & =0 \\
\rho\left[\frac{\partial \mathbf{v}}{\partial t}+(\mathbf{v} \cdot \nabla) \mathbf{v}\right] & =\rho \mathbf{f}-\nabla p+\nabla \cdot(\eta \nabla \mathbf{v})+\frac{1}{3} \nabla(\eta \nabla \cdot \mathbf{v}) \\
\rho c_{v}\left[\frac{\partial T}{\partial t}+(\mathbf{v} \cdot \nabla) T\right] & =\nabla \cdot(\kappa \nabla T)+\Phi-p \nabla \cdot \mathbf{v},
\end{aligned}
$$

where $\rho$ is density, $\mathbf{v}$ velocity, $\mathbf{f}$ body force acceleration, $p$ pressure, $\eta$ viscosity, $c_{v}$ heat capacity, $\kappa$ thermal conductivity, and $\Phi$ the dissipation function (i.e., viscous heating). Pressure-driven flow is related to the acceleration-driven case because a constant gradient of pressure will serve as a constant body force (acceleration), that is, the first two terms on the right hand side of Eq. (2) are equivalent at the hydrodynamic level. At the microscopic level the two driving forces are very different since an external force accelerates individual particles while a pressure gradient induces a collective flow due to a spatial variation of the particles. For accelerationdriven Poiseuille flow only the first two terms on the right hand side of Eq. (3) contribute but for pressure-driven flow there can also be cooling by expansion when the flow is compressible (i.e., when $\nabla \cdot \mathbf{v} \neq 0$ ).

In this paper the velocity, temperature, and pressure profiles are obtained from DSMC simulations for both acceleration-driven and pressure-driven Poiseuille flows and compared with numerical solutions of the Navier-Stokes equations. In Section 2 the numerical schemes for the Navier-Stokes solver and the DSMC simulations are described. Section 3 presents the results of the numerical investigations and in Section 4 a discussion of our results and a list of further directions are given.

\section{POISEUILLE FLOW}

In Poiseuille flow the fluid is confined between two rigid parallel plates that are stationary and act as thermal reservoirs. In the acceleration-driven case the boundary conditions are take as periodic in the flow direction and 
a constant body force is applied in this direction. In the pressure-driven case the boundary conditions are set to create a pressure gradient and no external field is applied.

The numerical solution of the compressible Navier-Stokes (NS) equations is obtained using a second-order Godunov method ${ }^{(13-15)}$ to evaluate the hyperbolic fluxes and a Crank-Nicolson scheme to treat the parabolic terms, which are approximated by standard finite difference methods. The computation of the hyperbolic flux terms using the second-order Godunov procedure is an explicit procedure so that the integration algorithm has a time step restriction based on CFL considerations of the Euler equations. The implicit discretization of the parabolic terms requires the solution of a nonlinear system of equations and this is done by the standard GaussSeidel method. The equations are solved in their conservative form using the temperature dependent transport coefficients for a hard sphere gas.

Direct Simulation Monte Carlo (DSMC) ${ }^{(16)}$ is a well-known algorithm for computing gas dynamics at the level of the Boltzmann equation. In DSMC, the state of the system is given by the positions and velocities of particles. In each time step, the particles are first moved as if they did not interact with each other. After moving the particles and imposing any boundary conditions, collisions are evaluated by a stochastic process, conserving momentum and energy and selecting the post-collision angles from their kinetic theory distributions. Being a stochastic algorithm, DSMC measurements have statistical variation but this variation is that of spontaneous fluctuations and so confidence intervals (error bars) may be evaluated using statistical mechanics. ${ }^{(17)}$

Although the geometry of the problem is simple, some care must be taken to formulate and implement boundary conditions that are equivalent in the NS and DSMC computations. The boundary conditions for the Navier-Stokes equations are: at the entrance $\left(x=-L_{x} / 2\right)$,

$$
\rho=\rho_{0}, \quad d \mathbf{v} / d x=0, \quad p=p_{+}
$$

and at the exit $\left(x=L_{x} / 2\right)$,

$$
d \rho / d x=0, \quad d \mathbf{v} / d x=0, \quad p=p_{-} .
$$

These boundary conditions were chosen in an effort to minimize the entrance and exit boundary layers, that is, mimic flow in a very long system. At the thermal walls $\left(y= \pm L_{y} / 2\right)$, the velocity and temperature boundary conditions are $\mathbf{v}=u_{\text {slip }} \hat{\mathbf{x}}$ and $T=T_{0}+T_{\text {jump }}$, where the slip and jump corrections for a fully accommodating surface are ${ }^{(18)}$

$$
u_{\text {slip }}=\alpha \lambda \frac{\partial u}{\partial y}, \quad T_{\text {jump }}=\beta \frac{2 \gamma}{\gamma+1} \frac{\lambda}{\operatorname{Pr}} \frac{\partial T}{\partial y},
$$


where $\lambda$ is the mean free path, $\gamma$ is the ratio of specific heats, and $\operatorname{Pr}$ is the Prandtl number. Ohwada et al. ${ }^{(19)}$ and Sone et al. ${ }^{(20)}$ predict the coefficients $\alpha=1.11$ and $\beta=1.13$, which is confirmed by DSMC simulations. ${ }^{(21)}$

In the NS computation, the inflow boundary conditions are imposed by setting the values of the grid points at the entrance as,

$$
\rho_{\text {in }}=\rho_{0}, \quad \mathbf{v}_{\text {in }}=\mathbf{v}_{\text {in }+1}, \quad p_{\text {in }}=p_{+},
$$

where $\mathbf{v}_{\mathrm{in}+1}$ is the velocity at the grid point immediately to the right of an entrance grid point. The outflow boundary conditions at the exit are imposed in a similar fashion

$$
\rho_{\text {out }}=\rho_{\text {out }-1}, \quad \mathbf{v}_{\text {out }}=\mathbf{v}_{\text {out }-1}, \quad p_{\text {out }}=p_{-} .
$$

The inflow and outflow boundary conditions for DSMC are imposed by refreshing the particles in the cells at the entrance and exit as if they were in contact with a reservoir. At the entrance, the number of particles in each cell is set according to the boundary condition $\rho=\rho_{0}$. The individual particle velocities are generated according to the Maxwell-Boltzmann distribution with a temperature $T=m p_{+} / \rho k$ (perfect gas) and with a mean velocity equal to that of the adjacent fluid cell, $\mathbf{v}_{\mathrm{in}+1}$. At the exit the number of particles in each cell is set equal to the number in the adjacent fluid cell so that $d \rho / d x=0$; the particle velocities are set as at the entrance but using the exit pressure $p_{-}$. To minimize fluctuations the fluid velocity used in setting the entrance and exit boundary cells is evaluated as a running average.

\section{NUMERICAL RESULTS}

The simulated fluid is a hard sphere gas with particle mass $m=1$ and diameter $d=1$. At the reference density of $\rho_{0}=1.21 \times 10^{-3}$, the mean free path is $\lambda_{0}=m\left(\sqrt{2} \pi \rho_{0} d^{2}\right)^{-1}=186$. The distance between the thermal walls is $L_{y}=10 \lambda_{0}$ and their temperature is $T_{0}=1$. The reference fluid speed is $u_{0}=\sqrt{2 k T_{0} / m}=1$ so Boltzmann's constant is taken as $k=1 / 2$. The reference sound speed is $c_{0}=\sqrt{\gamma k T_{0} / m}=0.91$ since $\gamma=5 / 3$ for a monatomic gas. The reference pressure is $p_{0}=\rho_{0} k T_{0} / m=6.05 \times 10^{-4}$. The acceleration and pressure gradient are chosen so that the flow will be sub-sonic, laminar and of similar magnitude in the two cases. Specifically, $\rho_{0} f=8.31 \times 10^{-8}$ for the acceleration-driven case and $d P / d x \approx 1.08 \times 10^{-7}$ for the pressuredriven case $\left(p_{+}=\frac{3}{2} p_{0}, p_{-}=\frac{1}{2} p_{0}, L_{x}=30 \lambda_{0}\right)$. In both cases the Knudsen number is $\mathrm{Kn}=\lambda / L_{y}=0.1$, the Mach number is $\mathrm{Ma} \approx 0.5$ so the Reynolds number $\mathrm{Re} \approx \mathrm{Ma} / \mathrm{Kn}$ is of order one. Computations were also performed 
for a variety of other Knudsen numbers, pressure ratios, aspect ratios, etc, and similar results were obtained. As expected, the DSMC and NS computations were in better agreement at smaller Knudsen number.

The code was first tested for the acceleration-driven case since, as described in Section 1, this scenario has received considerable attention in the literature. The results, shown in Figs. 1-3, are in agreement with earlier studies and clearly show that the solution of the Navier-Stokes equations qualitatively does not match the DSMC measurement. Namely, in DSMC one observes a non-constant pressure profile and a temperature dip in the center of the channel. On the other hand, the Navier-Stokes equations do accurately predict the velocity profile, which is nearly parabolic.

The results for the pressure-driven case are shown in Figs. 4-9. The comparison with the acceleration-driven case is best made by considering the profiles in the cross-stream directions measured at the center of the system (Figs. 4-6). As with the acceleration-driven case the Navier-Stokes equations are in qualitatively agreement with the DSMC measurement for the velocity profile (Fig. 4) but unlike before the temperature profile (Fig. 6) is also in qualitative agreement. In the acceleration-driven case $T>T_{0}$ due to viscous heating but in the pressure-driven case $T<T_{0}$ since the viscous heating is surpassed by the expansion cooling. Interestingly, the DSMC data indicate a reverse temperature jump at the wall, that is,

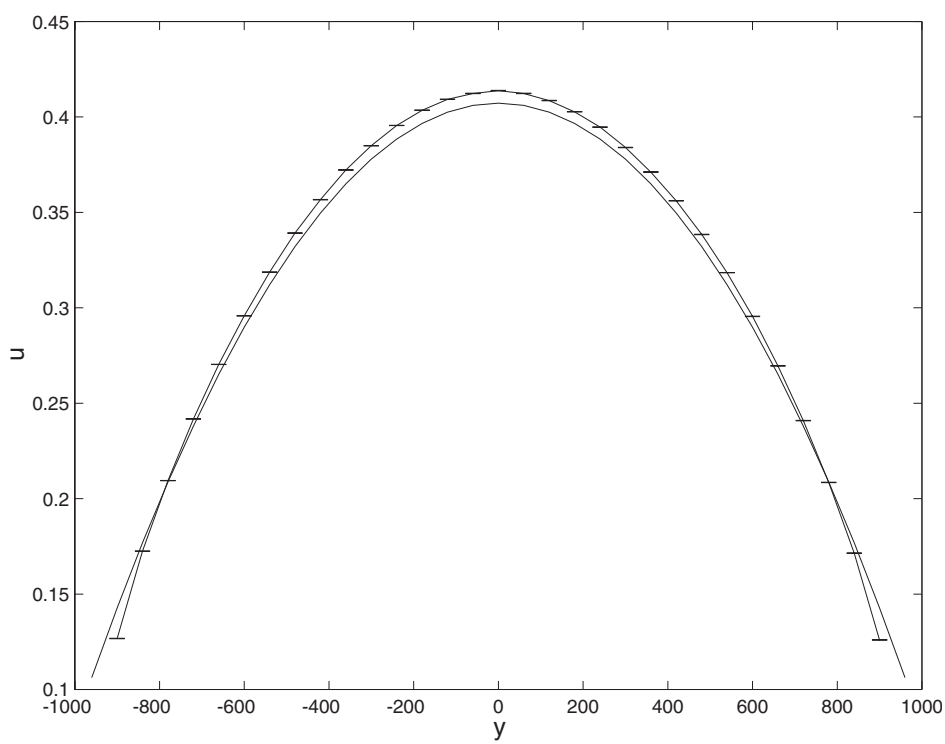

Fig. 1. Stationary velocity for acceleration-driven case; the curve with error bars is the DSMC result. 


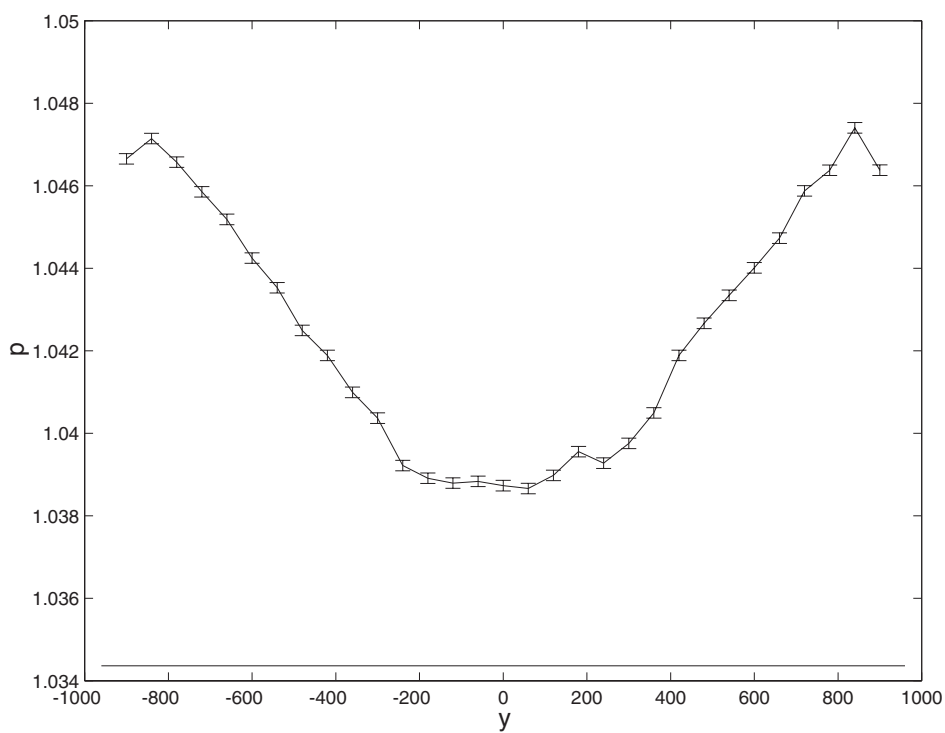

Fig. 2. Stationary pressure profile (normalized by $p_{0}$ ) for acceleration-driven case; the curve with error bars is the DSMC result.

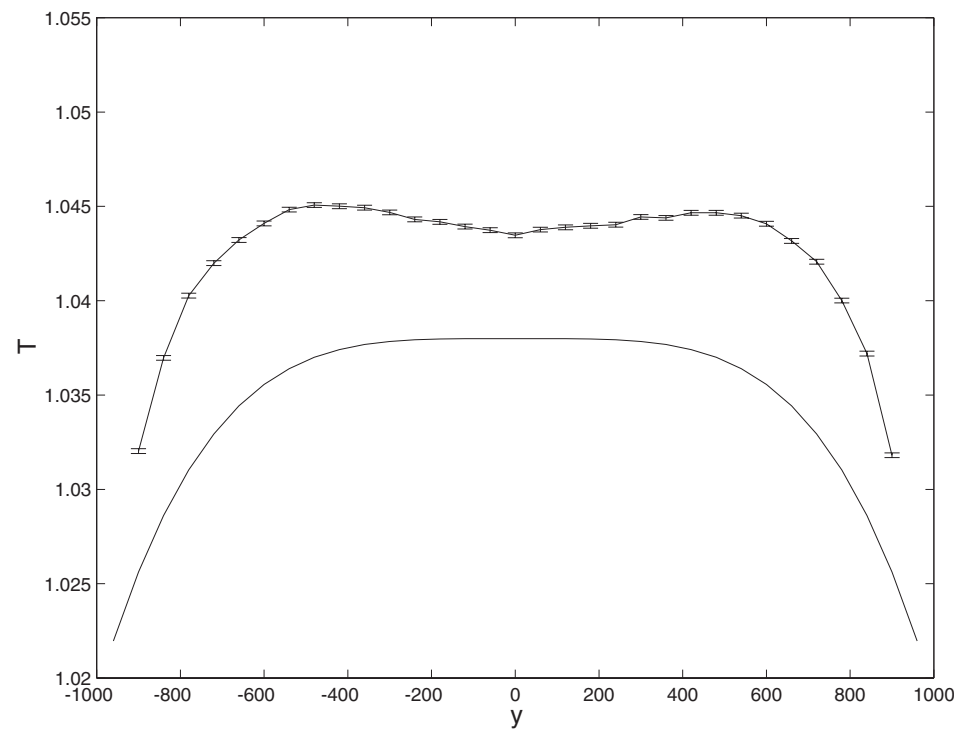

Fig. 3. Stationary temperature profile for acceleration-driven case; the curve with error bars is the DSMC result. 




Fig. 4. Stationary velocity profile for pressure-driven case; the curve with error bars is the DSMC result. The profiles are in the cross-stream direction ( $y$-direction) at the center of the system $(x=0)$.

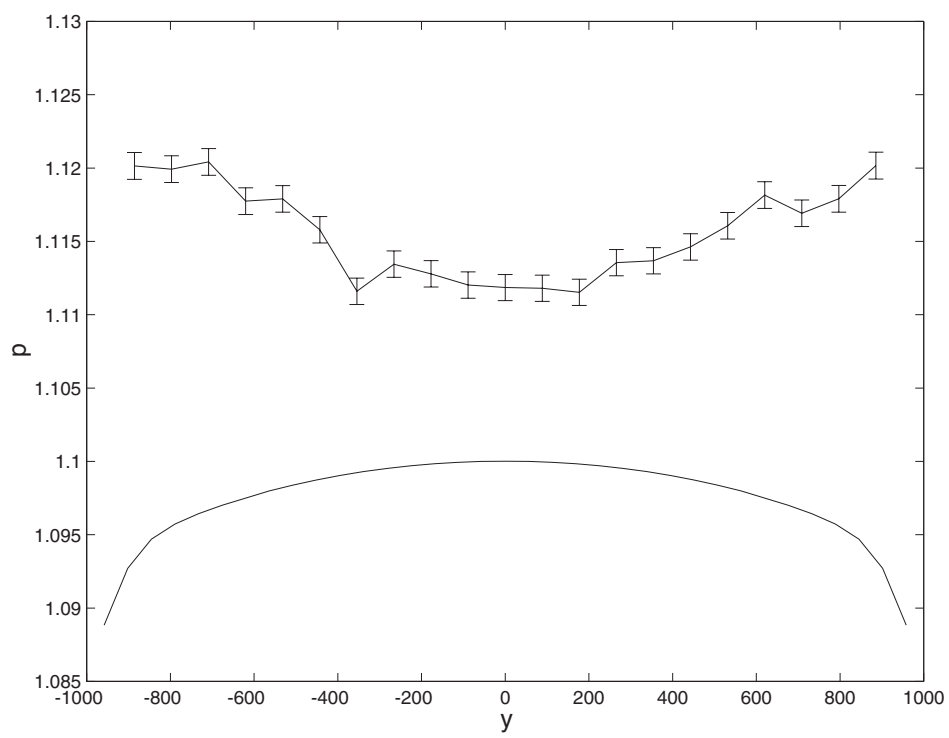

Fig. 5. Stationary pressure profile (normalized by $p_{0}$ ) for pressure-driven case; the curve with error bars is the DSMC result. The profiles are in the cross-stream direction ( $y$-direction) at the center of the system $(x=0)$. 


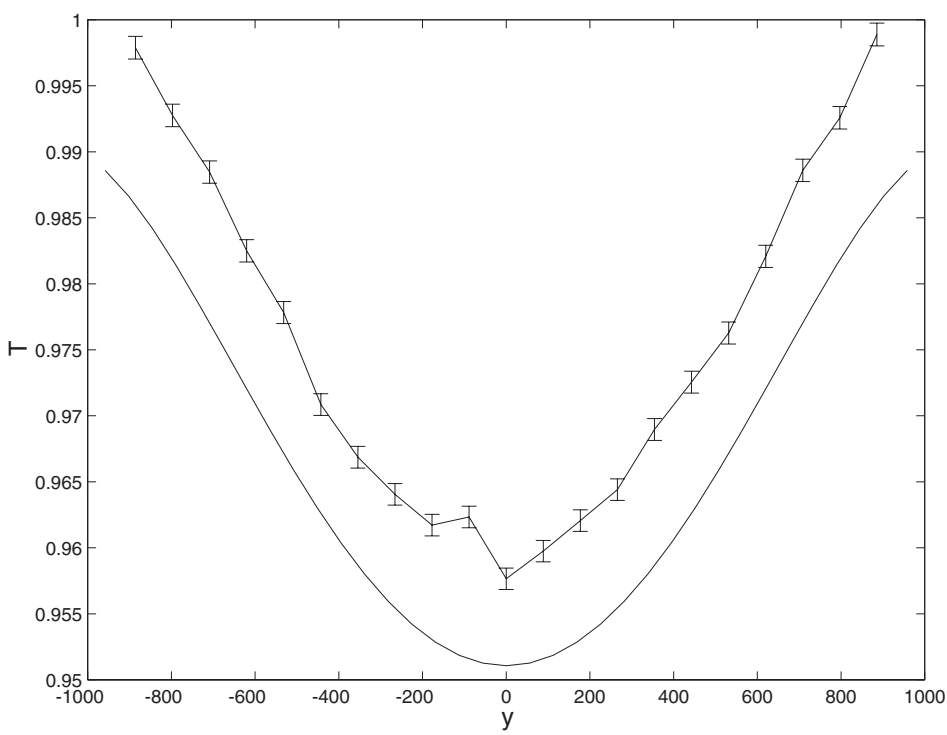

Fig. 6. Stationary temperature profile for pressure-driven case; the curve with error bars is the DSMC result. The profiles are in the cross-stream direction ( $y$-direction) at the center of the system $(x=0)$.

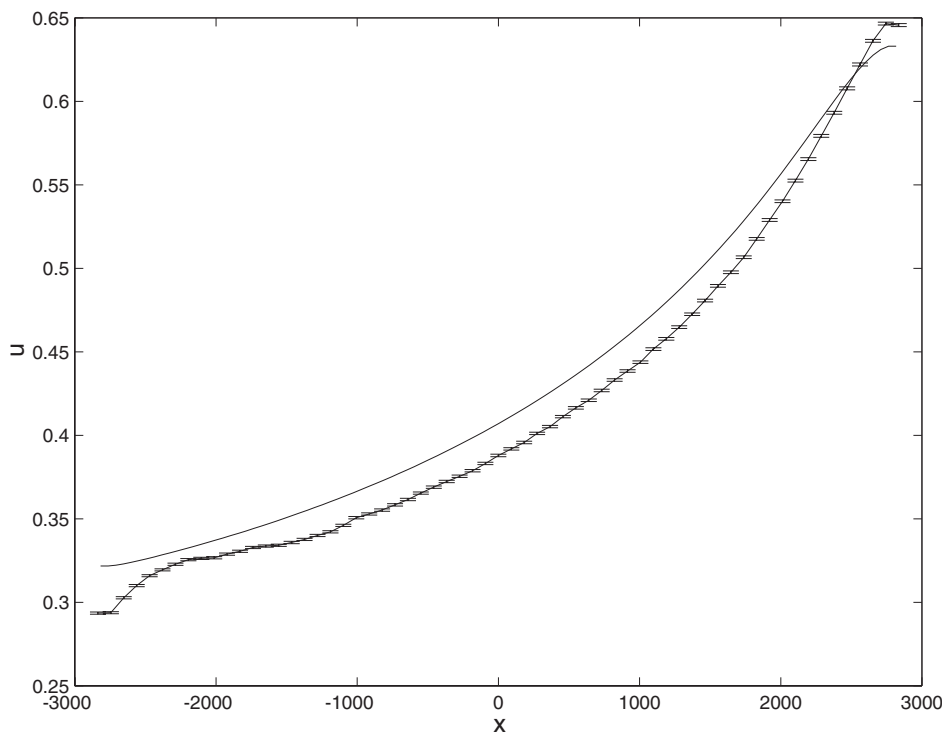

Fig. 7. Stationary velocity profile for pressure-driven case; the curve with error bars is the DSMC result. The profiles are in the stream-wise direction ( $x$-direction) down the center-line $(y=0)$. 


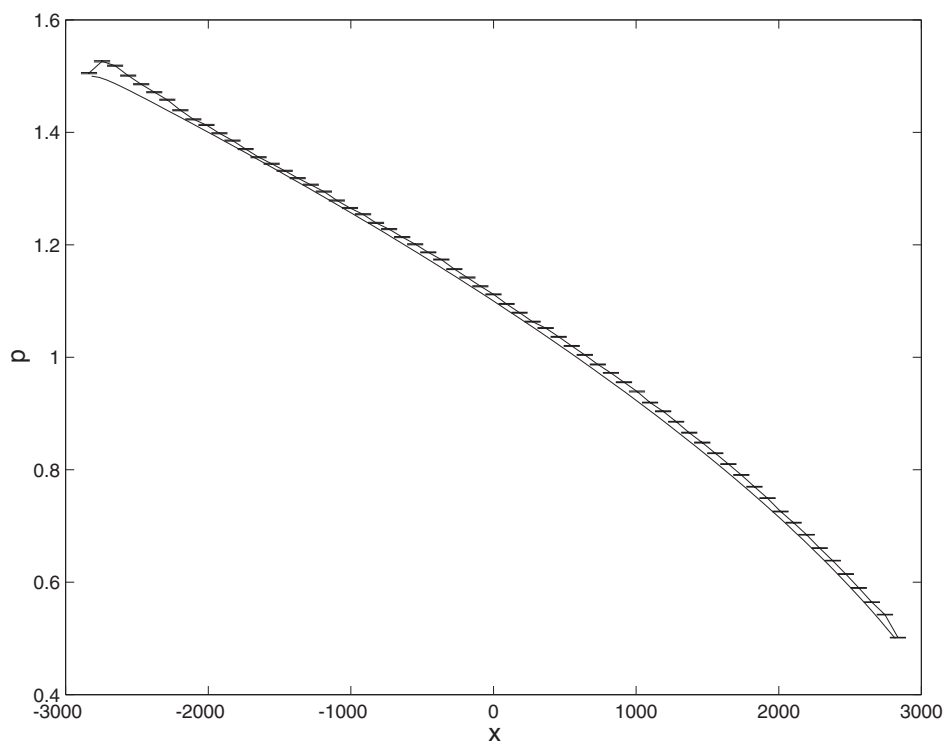

Fig. 8. Stationary pressure profile (normalized by $p_{0}$ ) for pressure-driven case; the curve with error bars is the DSMC result. The profiles are in the stream-wise direction ( $x$-direction) down the center-line $(y=0)$.

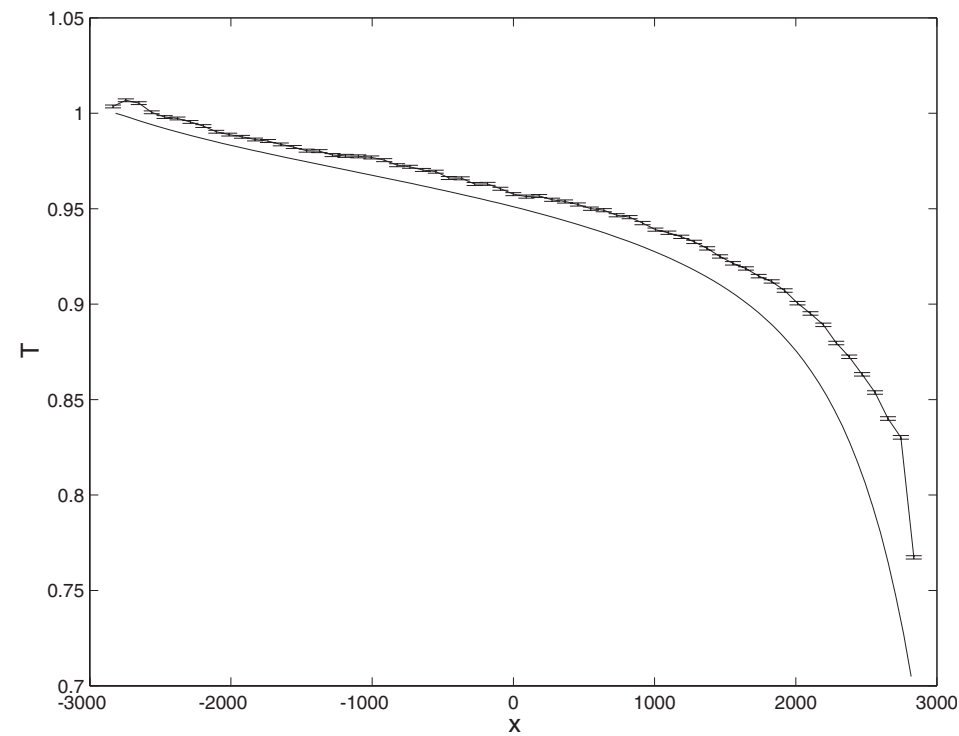

Fig. 9. Stationary temperature profile for pressure-driven case; the curve with error bars is the DSMC result. The profiles are in the stream-wise direction ( $x$-direction) down the center-line $(y=0)$. 
the temperature of the gas near the walls is slightly greater than $T_{0}$ though the temperature gradient normal to walls is negative. The NS and DSMC pressure profiles have opposite curvature (Fig. 5) but the magnitude of the pressure is nearly equal in the stream-wise direction (Fig. 8). The streamwise velocity and temperature profiles (Figs. 7 and 9) do not agree as well but this is not so surprising given that $\mathrm{Kn}=0.1$ and the aspect ratio is only $L_{x} / L_{y}=3$.

\section{CONCLUSIONS}

In this paper, plane Poiseuille flow is studied for acceleration-driven and pressure-driven conditions by comparing DSMC simulations with numerical solutions of the Navier-Stokes equations. In both acceleration and pressure-driven flows the velocity profile is approximately parabolic; the NS solution is in better agreement with the DSMC results in the former case. The qualitative difference observed in the temperature profile in the acceleration-driven case is not found in the pressure-driven case but the quantitative agreement is only fair. A concave pressure profile is observed in the DSMC data for both types of flow though NS give a strictly constant pressure in the acceleration-driven flow and a convex profile in the pressure-driven flow.

This investigation of pressure-driven Poiseuille flow is being extended in various directions. Some of the quantitative differences observed in the profiles are likely due to the influence of the entrance and exit conditions. These effects may be minimized by increasing the aspect ratio but then the pressure difference must be increased to maintain the same pressure gradient and the DSMC simulations become computationally more expensive. A better approach is to modify the entrance and exit reservoir conditions to better mimic the flow in a long channel. The quantitative and qualitative differences will also vary with Knudsen number, as has already been shown for acceleration-driven Poiseuille flow, and a similar analysis is underway for pressure-driven flow.

Besides velocity, temperature and pressure there are other quantities of interest, such as the various components of the stress tensor and heat flux, and a full analysis will be presented elsewhere. Finally, the DSMC results will be compared with kinetic theory (BGK approximation, moment methods, etc.) in cases where predictions are available.

\section{ACKNOWLEDGMENTS}

The authors wish to thank K. Aoki, G. Bird, N. Hadjiconstantinou, S. Hess, R. Hornung, M. Malek-Mansour, and S. Wijesinghe for helpful 
discussions. This work was performed under the auspices of the U.S. Department of Energy by University of California Lawrence Livermore National Laboratory under contract No. W-7405-Eng-48.

\section{REFERENCES}

1. J. R. Dorfman, An Introduction to Chaos in Nonequilibrium Statistical Mechanics (Cambridge University Press, Cambridge, 1999).

2. M. Mareschal, M. M. Mansour, A. Puhl, and E. Kestemont, Phys. Rev. Lett. 61:2550 (1988). A. Puhl, M. M. Mansour, and M. Mareschal, Phys. Rev. A 40:1999 (1989).

3. J. Koplik and J. R. Banavar, Ann. Rev. Fluid Mech. 27:257 (1995).

4. A. Santos, J. J. Brey, C. S. Kim, and J. W. Dufty, Phys. Rev. A 39:320 (1989). C. S. Kim, J. W. Dufty, A. Santos, and J. J. Brey, Phys. Rev. A 40:7165 (1989).

5. M. Tij and A. Santos, J. Statist. Phys. 76:1399 (1994).

6. M. M. Malek, F. Baras, and A. L. Garcia, Phys. A 240:255 (1997).

7. M. Tij, M. Sabbane, and A. Santos, Phys. Fluids 10:1021 (1998).

8. D. Risso and P. Cordero, Phys. Rev. E 58:546 (1998).

9. F. J. Uribe and A. L. Garcia, Phys. Rev. E, 60:4063 (1999)

10. S. Hess and M. Malek-Mansour, Phys. A 272:481 (1999).

11. K. Aoki, S. Takata, and T. Nakanishi, Phys. Rev. E 65:026315:1 (2002).

12. L. Landau and E. Lifshitz, Fluid Mechanics (Pergamon, London, 1959).

13. E. Toro, Riemann Solvers and Numerical Methods for Fluid Dynamics: A Practical Introduction (Springer-Verlag, Berlin/New York, 1999).

14. C. Laney, Computational Gasdynamics (Cambridge University Press, Cambridge/New York, New York, 1998).

15. M. Ben-Artzi and J. Falcovitz, J. of Comp. Phys. 55:1 (1984).

16. G. A. Bird, Molecular Gas Dynamics (Clarendon, Oxford, 1976). G. A. Bird, Molecular Gas Dynamics and the Direct Simulation of Gas Flows (Clarendon, Oxford, 1994).

17. N. Hadjiconstantinou and A. Garcia, Proceedings of the First MIT Conference on Computational Fluid and Solid Mechanics, Vol. 853 (Elsevier, 2001).

18. C. Cercignani, Theory and Application of the Boltzmann Equation (Scottish Academic Press, Edinburgh, 1975).

19. T. Ohwada, Y. Sone, and K. Aoki, Phys. Fluids A 1:1588 (1989).

20. Y. Sone, T. Ohwada, and K. Aoki, Phys. Fluids A 1:363 (1988).

21. S. Wijesinghe, N. Hadjicontantinou, Proceedings of the First MIT Conference on Computational Fluid and Solid Mechanics, Vol. 2 (Elsevier, 2001), p. 1019. 